\title{
B-Cell Receptor-Associated Protein 31 Regulates the Expression of Valosin- Containing Protein Through Elf2
}

\author{
Cong-cong Jia ${ }^{a}$ Juan Du ${ }^{a}$ Xia Liu ${ }^{a}$ Rui Jianga Yongye Huang ${ }^{a}$ \\ Tianyi Wang ${ }^{a}$ Yue Hou ${ }^{a}$ Bing Wanga,b

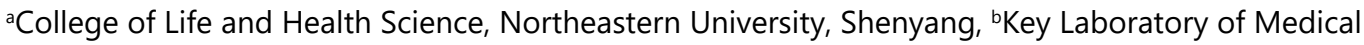 \\ Image Computing, Liaoning Provine, Shenyang, China
}

\section{Key Words}

Bap31 • VCP • CFTR $\triangle \mathrm{F} 508 \cdot \mathrm{ERAD} \cdot$ Elf2 $・$ CNS diseases

\begin{abstract}
Background/Aims: B-cell receptor-associated protein 31 (Bap31) is an evolutionarily conserved, ubiquitously expressed, polytopic integral membrane protein in the endoplasmic reticulum (ER) that is involved in the regulation of apoptosis, protein transport and degradation. Patients with Bap31 mutations exhibit symptoms similar to those exhibited by patients with central nervous system (CNS) diseases, such as deafness, dystonia, and intellectual disability. The present study aimed to investigate the function of Bap31 in CNS diseases by identifying a CNS disease-related gene regulated by Bap31 and exploring the underlying molecular mechanism. Methods: ShRNA-Bap31 and siRNA-Bap31 were used to knockdown Bap31 in $\mathrm{N}_{2}$ a cells, and real-time PCR was performed to detect the mRNA levels of genes involved in CNS diseases. Western blot analyses were used to examine the protein levels of the candidate gene (valosin-containing protein, VCP) both in vivo and in vitro. The functions of Bap31 and VCP in mediating the degradation of the hyper-unstable mutant of cystic fibrosis trans-membrane conductance regulator (CFTR $\triangle F 508$ ) were studied. Moreover, real-time PCR, Western blot and dual luciferase reporter analyses were conducted to investigate the molecular mechanism by which Bap31 regulates the expression levels of VCP. Results: VCP was identified as a candidate gene based on its differential expression in $\mathrm{N}_{2}$ a cells following both shRNA- and siRNAmediated knockdown of Bap31. Both the mRNA and protein levels of VCP were regulated by Bap31 in vivo and in vitro. In the ER-associated degradation (ERAD) pathway, Bap31 also regulated VCP expression and caused differences in the binding quantities of CFTR $\triangle F 508$ and VCP. Furthermore, a transcription factor of VCP (E74-like factor 2, Elf2) was regulated by Bap31, and Elf2 mediated the changes in VCP transcription and expression in cells with altered Bap31 expression. Conclusion: These results indicate that Bap31 regulates the expression of VCP possibly via Elf2 and support the potential molecular function of Bap31 in CNS diseases.

Dr. Bing Wang

and Dr. Yue Hou

Institute of Biochemistry and Molecular Biology; College of Life and Health Science Northeastern University, Nr. 500 Zhihui Street, Hunnan Xinqu, Shenyang 110000 (China) Tel. +862483656125, E-Mail wangbing@mail.neu.edu.cn; houyue@mail.neu.edu.cn
\end{abstract}

KARGER 


\section{Cellular Physiology Cell Physiol Biochem 2018;51:1799-1814

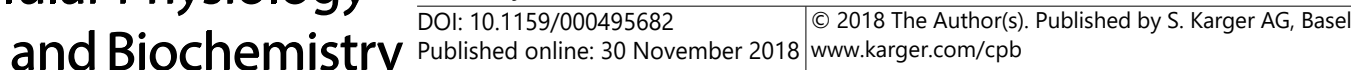 Jia et al.: Bap31 Regulates VCP via Elf2}

\section{Introduction}

B-cell receptor-associated protein 31 (Bap31) is a $28 \mathrm{kDa}(\mathrm{p} 28)$ polytopic integral protein located on the membrane of the endoplasmic reticulum (ER). Bap31 contains a hydrophobic N-terminus and an alpha-helical C-terminus that ends with a KKXX motif [1]. The cytosolic C-terminal domain can be cleaved by caspase 8 to generate a pro-apoptotic $20 \mathrm{kDa}$ fragment (p20) [2]. Bap31 is a sorting protein that controls the transport of certain membrane proteins from the ER to the Golgi apparatus [3]. Our previous studies have indicated that Bap31 may perform an important function in T-cell activation by regulating T-cell receptor (TCR) signaling [4] and revealed for the first time that Bap31 is involved in the ER-associated protein degradation (ERAD) pathway [5]. Moreover, Bap31 participates in the regulation of neuronal survival, and the loss of Bap31 function induces a severe X-linked phenotype with congenital microcephaly or sensory neural deafness in humans $[6,7]$.

Many proteins can be degraded by the ubiquitin-proteasome system via the ERAD pathway. Valosin-containing protein (VCP), which is also known as CDC48 in yeast, is an oligomeric ATPase composed of one $\mathrm{N}$ - terminal domain and two ATPase domains (D1 and D2) $[8,9]$. VCP promotes the degradation of unfolded or misfolded proteins to maintain the quality of the ER by interacting with Gp78 [10-12]. In addition, as an ATPase, VCP can maintain neuronal survival and protect against neurodegeneration [13]. Many diseases, including frontotemporal dementia 1, amyotrophic lateral sclerosis and inclusion body myopathy with early-onset Paget's disease, have been shown to be associated with VCP [14]. Moreover, research has shown that high levels of VCP expression in cancer cells are correlated with an increased recurrence rate $[15,16]$. Transcription factors, such as E74-like factor 2 (Elf2), which is an ETS family transcription factor, can bind the 5' flanking region of the VCP promoter, which contains consensus binding sites, to increase the expression of VCP [17].

Cacciagli et al. reported that people with mutations in Bap31, including seven individuals from three families, suffer from motor and intellectual disabilities, dystonia, sensory neural deafness and white-matter changes. Although this phenotype is defined as an X-linked syndrome, these phenotypes share some similarities with central nervous system (CNS) diseases [7]. However, there are currently no reports regarding the regulation of genes involved in CNS diseases by Bap31. Therefore, in the present study, we investigated the potential molecular mechanisms underlying the role of Bap31 in CNS diseases by searching for related genes that are regulated by Bap31. The results showed that one of the Bap31regulated genes is VCP. Bap31 regulates the expression of VCP and modulates the ERAD process in which VCP is involved. In addition, we found that Bap31 regulates VCP expression by affecting the expression levels of its transcription factor, Elf2. Altogether, our findings highlight the functional role of Bap31 in CNS diseases.

\section{Materials and Methods}

\section{Cell lines and cell culture}

Mouse neuroblastoma $\left(\mathrm{N}_{2} \mathrm{a}\right)$ cells and baby hamster kidney (BHK) cells were cultured in Dulbecco's modified Eagle medium (DMEM, Gibco, CA, USA) with 10\% fetal bovine serum (Tbd Science Technology, Tianjin, China) and $1 \%$ penicillin-streptomycin solution (Biological Industries, CT, USA) at $37{ }^{\circ} \mathrm{C}$ in $5 \% \mathrm{CO}_{2}$. BHK cells stably expressing CFTR $\Delta$ F508-HA were selected using $500 \mu \mathrm{M}$ methotrexate cultured in DMEM medium under normal conditions. 


\section{Cellular Physiology Cell Physiol Biochem 2018;51:1799-1814

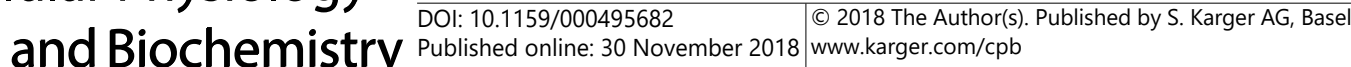

Jia et al.: Bap31 Regulates VCP via Elf2

Antibodies and plasmids

The following antibodies were used in this study: anti-mouse VCP, anti-mouse $\beta$-actin, anti-mouse HA tag (Abcam, MA, USA), anti-rabbit Elf2 (Abisin, Shanghai, China), anti-rabbit Derlin1 (Sigma-Aldrich, MO, USA), and secondary anti-mouse or anti-rabbit antibodies (ThermoFisher Scientific, MA, USA).

VCP, Bap31, Derlin1 and Elf2 cDNAs were individually subcloned into pcDNA3.1(-) vectors (Promega, WI, USA). To construct the reporter plasmid, polymerase chain reaction (PCR) was used to amplify a fragment containing a segment of the VCP promoter with Elf2 binding sites. The fragment was subcloned into a pGL3-basic vector (Promega). The primers used to construct the plasmids are shown in Table S1 (Sangon Biological Engineering, Shanghai, China). For all supplemental material see www.karger.com/ 10.1159/000495682/.

\section{Animal models}

To generate mice with a Bap31 knockout specifically in the hippocampus, we constructed a targeting vector containing intron 3 of Bap31 flanked by two loxP sites. The neomycin resistant gene cassette located within the loxP sites was flanked by two flippase (Flp) recognition target (FRT) sites. The construct was electroporated into embryonic stem (ES) cells from C57BL/6 mice. Male chimeric mice were bred with Flp enzyme female transgenic mice to detect the expression of the neomycin gene. Then, the Bap31 ${ }^{\mathrm{f} / \mathrm{+}}$ mice were bred with Camk2a-cre transgenic mice to generate the Camk2a-cre Bap31 ${ }^{\mathrm{f} / \mathrm{f}}$, Camk2a-cre Bap31 ${ }^{\mathrm{f} /+}$ and Bap31 $1^{\mathrm{f} / \mathrm{f}}$ mice. The same-gender littermates of the Camk2a-cre Bap31 $1^{\mathrm{f} / \mathrm{f}}$ and Bap31 ${ }^{\mathrm{f} / \mathrm{f}}$ mice were used in this study. The mice were housed under standard conditions with a 12-hrs light/12-hrs dark cycle and free access to food and water. All animal use procedures were planned and performed in compliance with the Regulations of Experimental Animal Administration issued by the State Committee of Science and Technology of the People's Republic of China. The experiments were performed under the approval of the Committee of Experimental Animal Administration of the University.

\section{Transfection}

For the transfection, $3 \times 10^{5}$ cells were seeded in a $60-\mathrm{mm}$ dish. At $12 \mathrm{hrs}$ after seeding, the cells were transfected with a pL/shRNA/green fluorescent protein (GFP)-mouse-Bap31 construct (Novobio Technology, Shanghai, China) according to a multiplicity of infection (MOI) of 10. Cells transfected with $\mathrm{pL} / \mathrm{shRNA} / \mathrm{GFP}$ were used as control cells. Then, $10 \mu \mathrm{g} / \mathrm{ml}$ blasticidin and the limited dilution method were used to obtain the monoclonal cell strains.

A total of $3 \times 10^{5}$ cells were transfected with mixed siRNA (50 nM) and/or plasmids ( $2 \mu \mathrm{g}$ ) using Lipofectamine 3000 transfection reagent (Invitrogen, CA, USA) according to the manufacturer's instructions. The negative siRNA and/or pcDNA3.1(-) vector were used to generate the control cells. After transfection for $24 \mathrm{hrs}$, the same transfection was repeated. Real-time PCR and Western blot analyses were used to detect the transcription and expression levels of the interested genes after the first transfection for $72 \mathrm{hrs}$. The sequences of the siRNA used in this study were as follows: siRNA-VCP, 5'-gagagcaaccuucguaa-3', 5'-gcuacaggacgcuuggaaa-3', and 5'-gaauagaguuguucggaauuu-3' [18]; siRNABap31, 5'-ccucuacgcagaggucutt-3', 5'-ccauggagcacuuccacatt-3', and 5'-ggcuaugcagaagcaguctt-3'; siRNAElf2, 5'-gcugaagcccugcuucauatt-3', 5'-gcagucaccaguuuccaautt-3', and 5'-gcuagugacgugugagaaatt-3'; siRNA-Derlin1, 5'-gcgagcugcugaucagaatt-3', 5'-ggacuugggaggaaggaatt-3', and 5'-cugguucggaacgcgatt-3' (GenePharma, Shanghai, China).

\section{RNA extraction and real-time PCR}

The total RNA was isolated from the samples using TRIzol reagent (Ambion, CA, USA), and $2 \mu \mathrm{g}$ total RNA were used for the cDNA synthesis (Promega) according to the manufacturer's instructions. The mRNA levels of the genes were analyzed by real-time PCR reagents (Promega) with a CFX96 Touch ${ }^{\text {TM }}$ Real-time PCR Detection System (Bio-Rad Laboratories, CA, USA). The experiments were repeated three times with three replicates per detection. The results were analyzed according to the following formula: $2^{-\Delta \Delta C t}\left(\Delta \Delta \mathrm{Ct}=\left[\left(\mathrm{Ct}_{\text {target }}\right.\right.\right.$ gene $\left.\left.\left.-\mathrm{Ct}_{\mathrm{GAPDH}}\right)_{\text {sample }}-\left(\mathrm{Ct}_{\text {target gene }}-\mathrm{Ct}_{\mathrm{GAPDH}}\right)_{\text {control }}\right]\right)$ [19]. The primers used for the real-time PCR assay are listed in Table S2 (Sangon Biological Engineering). 


\section{Cellular Physiology Cell Physiol Biochem 2018:51:1799-1814 and Biochemistry \begin{tabular}{l|l} 
DOI: 10.1159/000495682 \\
Published online: 30 November 2018
\end{tabular} \\ Jia et al.: Bap31 Regulates VCP via Elf2}

\section{Western blot}

For the Western blot analysis of protein expression, the cells were resuspended in RIPA buffer [4] at $4{ }^{\circ} \mathrm{C}$ for $30 \mathrm{~min}$ and then centrifuged at $12,000 \mathrm{rpm}$ for $15 \mathrm{~min}$. Then, $5 \times$ sodium dodecyl sulfate (SDS) sample buffer was added to the supernatant, and the sample was boiled. The lysates were separated by $12 \%$ SDS-polyacrylamide gel electrophoresis (PAGE) and transferred onto polyvinylidene difluoride (PVDF) membranes (Millipore, MA, USA). After blocking with 5\% skim milk at room temperature for $1 \mathrm{hr}$, the membranes were incubated with the primary antibodies overnight at $4{ }^{\circ} \mathrm{C}$, washed with Tris-buffered saline containing Tween-20 (TBST) and incubated with the secondary antibodies. For the visualization of protein bands, the membranes were developed using an enhanced chemiluminescence kit (Tanon, Shanghai, China), and the resultant bands were analyzed with a ChemiDocTM XRS+ system (Bio-Rad Laboratories, CA, USA). All immunoblotting analyses were repeated at least three times, and the same results were obtained using Image Lab software (Bio-Rad). The band intensities of the target proteins were calculated and normalized to that of $\beta$-actin.

\section{Coimmunoprecipitation}

For the coimmunoprecipitation experiments, the cells were lysed in Chaps buffer or NP40 buffer [5]. After preclearing for 30 min with protein G Sepharose (Invitrogen), the lysates were incubated with antibodies for $2 \mathrm{hrs}$ at $4{ }^{\circ} \mathrm{C}$. The complexes bound to protein G Sepharose were recovered, washed three times with lysis buffer and eluted in SDS sample buffer. All samples were analyzed by Western blot analyses.

\section{Dual-luciferase assay}

After $\mathrm{N}_{2}$ a cells were transfected with the siRNA or the overexpression plasmids for $48 \mathrm{hrs}$, the cells were cotransfected with a luciferase reporter plasmid containing the VCP promoter and phRL-SV40 vector (the transfection efficiency control) with Lipofectamine 3000 for 48 hrs. The luciferase activities were detected using a dual luciferase assay kit (Beyotime, Shanghai, China) and determined with a plate reader (BioTek, VT, USA). The values of the VCP promoter luciferase reporter plasmid were normalized to those of the phRL-SV40 vector.

\section{Cell viability}

Cells at a density of $1 \times 10^{4}$ cells/well were seeded in a 96-well plate, incubated with the VCP inhibitor $\mathrm{N}_{2}, \mathrm{~N}_{4}$-dibenzylquinazoline-2, 4-diamine (DBeQ) for the indicated time and treated with $5 \mathrm{mg} / \mathrm{mL} \mathrm{MTT}$ (Sigma-Aldrich) at $37^{\circ} \mathrm{C}$ for $4 \mathrm{hrs}$. The supernatant was removed, and $150 \mu \mathrm{L}$ dimethyl sulfoxide (DMSO, Sigma-Aldrich) were added. The absorbance values were determined by a colorimetric assay using a plate reader (BioTek) at $490 \mathrm{~nm}$.

\section{Flow cytometry and immunofluorescence}

BHK cells stably expressing CFTR $\mathrm{F} 508$-HA were first fixed in 2\% paraformaldehyde for $15 \mathrm{~min}$ and then stained with an anti-HA antibody, followed by a fluorescein-isothiocyanate (FITC)-conjugated secondary antibody, for detection by flow cytometry using a Becton Dickinson fluorescence-activated cell sorter (FACS) system (FACSCalibur, Becton Dickinson, NJ, USA). For the immunofluorescence analysis, the same cell processing procedure used for flow cytometry analysis was applied, and nuclei were identified by staining with Hoechst 33342 (Sigma-Aldrich). The fluorescence images were taken under a confocal laser scanning microscope (Leica, IL, USA).

\section{Statistical analysis}

All data are expressed as the mean \pm standard error of the mean (SEM), and significant differences between groups were identified by independent sample Student's t-tests. All statistical procedures were performed using SPSS 17.0 software for Windows (SPSS, Inc., IL, USA). 


\section{Results}

Bap31 regulates the mRNA levels of genes involved in CNS diseases

Bap31 is abundantly expressed in various cell types, including neurons. To investigate the molecular functions of Bap31 in CNS diseases, we first constructed the shRNABap31 plasmid and then transfected the shRNABap31 plasmid or control plasmid into $\mathrm{N}_{2}$ a cells. The finite dilution method was used to obtain a monoclonal cell strain with reduced Bap31 expression (shRNA-Bap31). The realtime PCR analysis of the mRNA obtained from the shRNA-Bap31-transfected cell lines revealed that the expression levels of Bap31 were reduced to $17.3 \%$ compared with those in the control cells (Fig. 1A). Moreover, the Bap31 protein expression level in these cells was $10 \%$ of that in the control cells (Fig. 1B). Bayes et al. explored the postsynaptic density in the human neocortex (hPSD) and identified 1, 461 proteins. The analysis of the results indicated that 95 CNS diseases are caused by 83 genes [20]. To further understand the role of Bap31 in CNS diseases, we performed real-time PCR experiments to identify Bap31-regulated genes that are involved in CNS diseases using shRNA-Bap31transfected cells. According to the results shown in Fig. 1, the expression levels of 48 genes were significantly altered by the stable knockdown of

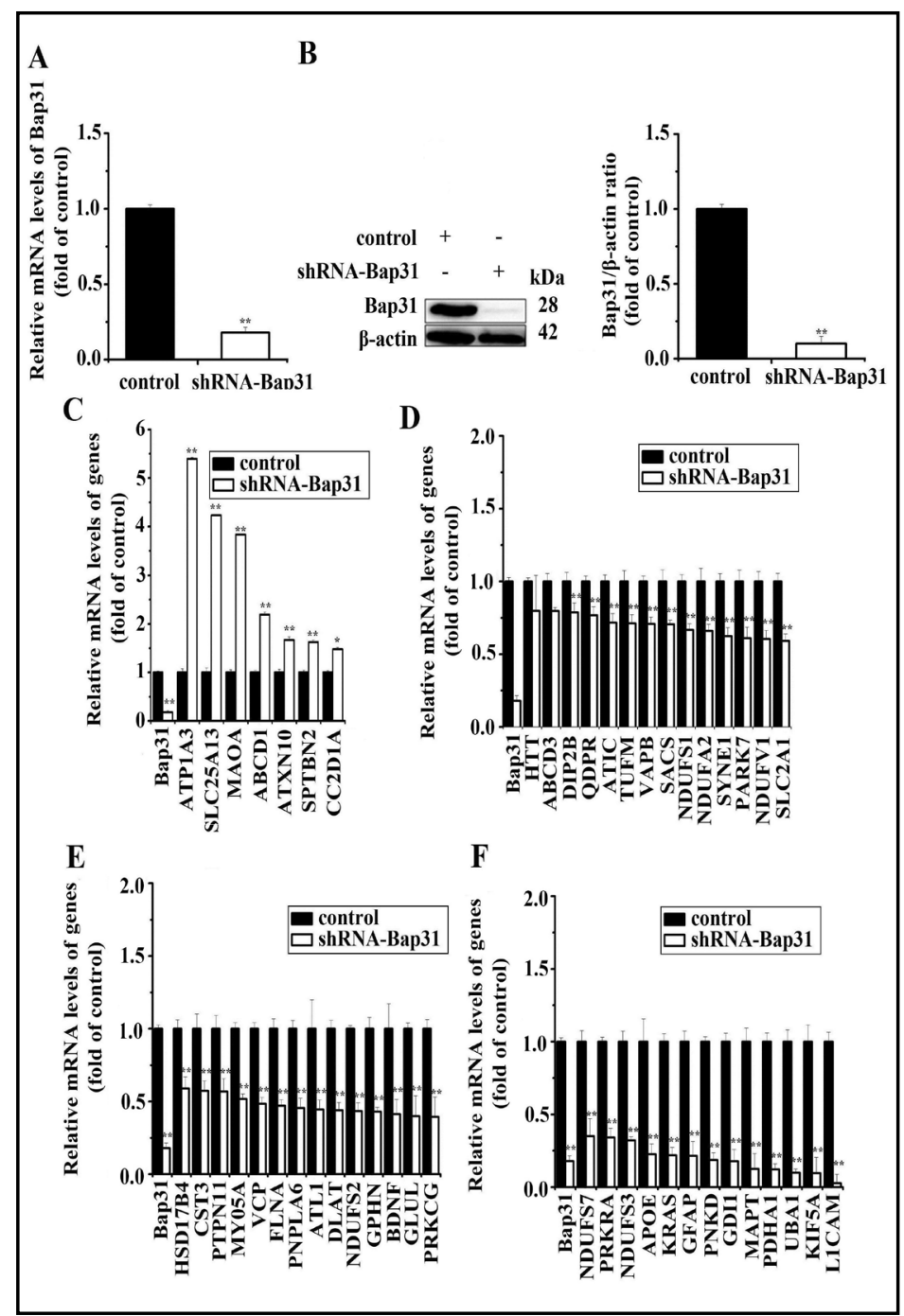

Fig. 1. Bap31 regulates the mRNA levels of genes involved in CNS diseases in shRNA-Bap31-transfected $\mathrm{N}_{2}$ a cells. Real-time PCR (A) and Western blot (B) analyses of Bap31 in stable Bap31 knockdown cells (shRNA-Bap31, $\mathrm{N}_{2}$ a cells transfected with pL/shRNA/GFPmouse-Bap31) and cells transfected with pL/shRNA/GFP as a control. Histograms showing the relative change in the Bap31 levels in shRNABap31-transfected cells and control cells. Real-time PCR analysis of the upregulated (C) and downregulated (D, E and F) genes involved in CNS diseases in shRNA-Bap31-transfected cells compared with those in the control cells. $p^{*}$ vs control $<0.05, p^{* *}$ vs control $<0.01$.

Table 1. Statistical table of the genes involved in CNS diseases identified in the Bap31 knockdown cells

\begin{tabular}{lccc}
\hline Groups & Upregulated & Downregulated & Total \\
\hline shRNA-Bap31 & 7 & 41 & 48 \\
siRNA-Bap31-48 h & 6 & 15 & 21 \\
siRNA-Bap31-72 h & 5 & 21 & 26 \\
\hline
\end{tabular}


Bap31, including 7 genes that were upregulated (Fig. 1C) and 41 genes that were downregulated (Fig. 1DF, Table 1). These results demonstrate that Bap31 regulates the mRNA levels of some genes involved in CNS diseases.

To avoid the potential confounding effects of an abnormal steady state within cells with alterations in many genes, we used siRNA-mediated depletion of Bap31 to study the effects of transient Bap31 knockdown on the expression of CNS diseaserelated genes. In the $\mathrm{N}_{2} \mathrm{a}$ cells treated with siRNABap31 for 48 or 72 hrs, the Bap31 mRNA levels were $31 \%$ and $40 \%$ of the levels observed in the control cells (Fig. 2A), and the corresponding protein levels of Bap31 were 28\% and $32 \%$ of those observed in the control cells (Fig. 2B). These results indicate that cells disturbed by siRNA-Bap31 could be used to study the expression levels of genes regulated by Bap31.

The detection of the mRNA levels of CNS diseaserelated genes in $\mathrm{N}_{2}$ a cells that had been transfected with siRNA-Bap31 for 48 hrs revealed 6 genes that were upregulated (Fig. 2C) and 15 genes that were downregulated (Fig. 2D). Thus, 21 genes showed differential expression following the transient Bap31 knockdown. Considering that this number of differentially expressed genes is less than half of that observed following stable Bap31 knockdown, we repeated the analysis of the $\mathrm{N}_{2}$ a cells transfected with siRNA-Bap31 for $72 \mathrm{hrs}$ and identified 5 genes that were upregulated (Fig. 2E) and 21 genes that were downregulated (Fig. 2F and $2 \mathrm{G}$ ). A total of 26 differentially expressed genes were identified

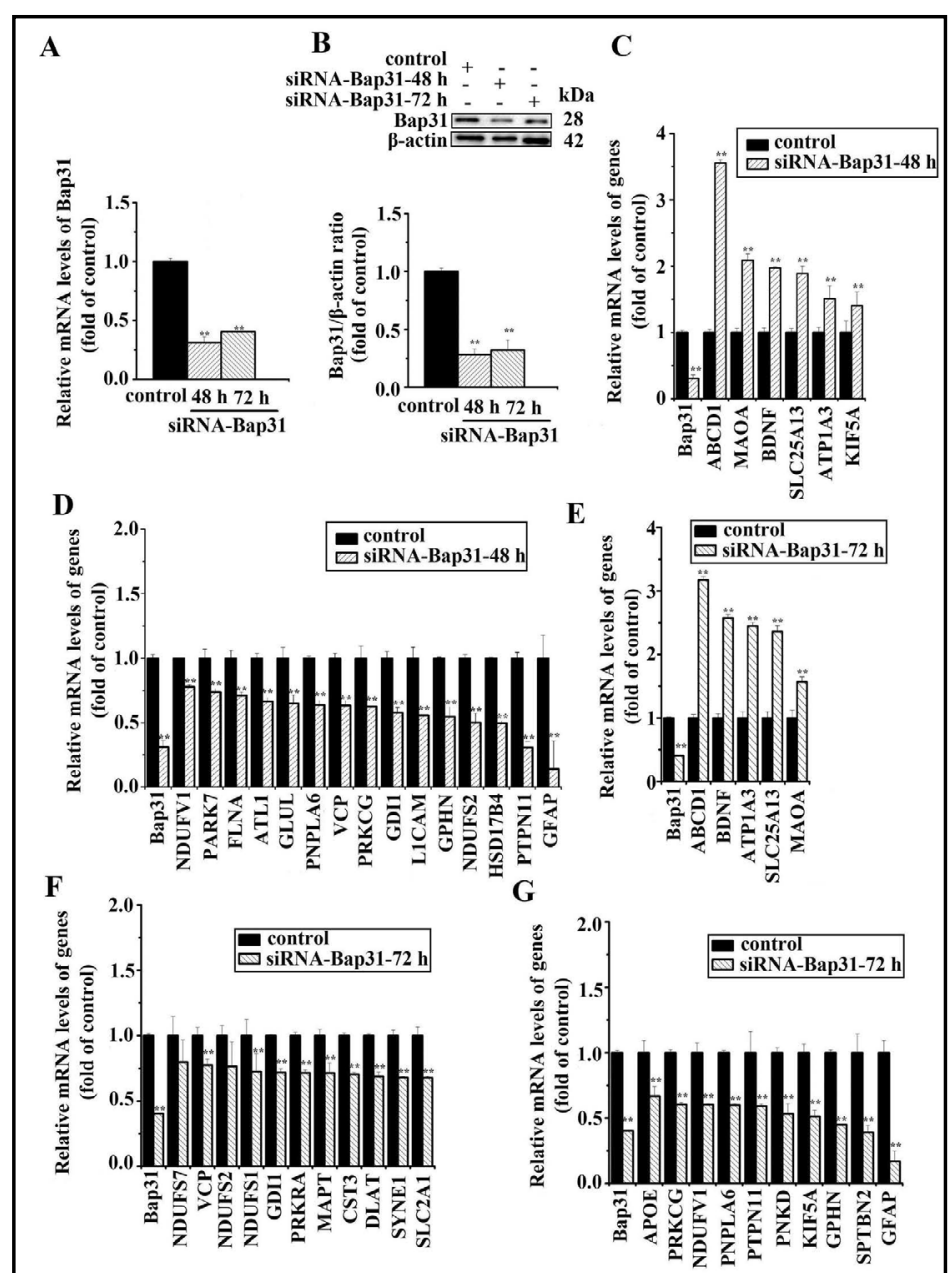

Fig. 2. Bap31 regulates the mRNA levels of genes involved in CNS diseases in $\mathrm{N}_{2}$ a cells transfected with siRNA-Bap31. Real-time PCR (A) and Western blot (B) analyses of Bap31 in $\mathrm{N}_{2}$ a cells transfected with siRNA-Bap31 for 48 and 72 hrs and cells transfected with negative siRNA as a control. Histograms showing the relative changes in the Bap31 levels in siRNA-Bap31-transfected cells and control cells. Realtime PCR analysis of the upregulated (C, E) and downregulated (D, F and $G$ ) genes involved in CNS diseases in $\mathrm{N}_{2}$ a cells transfected with siRNA-Bap31 for 48 and 72 hrs compared with those in the control cells. $p^{*}$ vs control $<0.05, p^{* *}$ vs control $<0.01$.

Table 2. Upregulated and downregulated genes involved in CNS diseases in the different Bap31 knockdown cells

\begin{tabular}{lcc}
\hline Groups & shRNA-Bap31 & siRNA-Bap31 \\
\hline upregulated & $A B C D 1$, ATP1A3, MAOA, SLC25A13 \\
downregulated & GDI1, GFAP, GPHN, NDUFS2, NDUFV1, PNPLA6, PRKCG, PTPN11, VCP
\end{tabular}

KARGER 
in $\mathrm{N}_{2}$ a cells (Table 1). These results further imply that Bap31 regulates the expression of genes that are associated with CNS diseases.

By comparing the genes that were differentially expressed in both the shRNA-Bap31- and siRNABap31-transfected cells, we identified 4 upregulated genes (ABCD1, ATP1A3, $M A O A$ and SLC25A13) and 9 downregulated genes (GDI1, GFAP, GPHN, NDUFS2, NDUFV1, PNPLA6, PRKCG, $P T P N 11$ and $V C P$, Table 2). The results demonstrate that these 13 genes are more likely to be regulated by Bap31 than other genes.

Bap31 regulates the protein levels of VCP in vitro

Among the 13 genes that were differentially expressed in the Bap31 knockdown cells, VCP is of particular interest in the present study because several reports have linked VCP to CNS diseases, such as Paget's disease of bone,

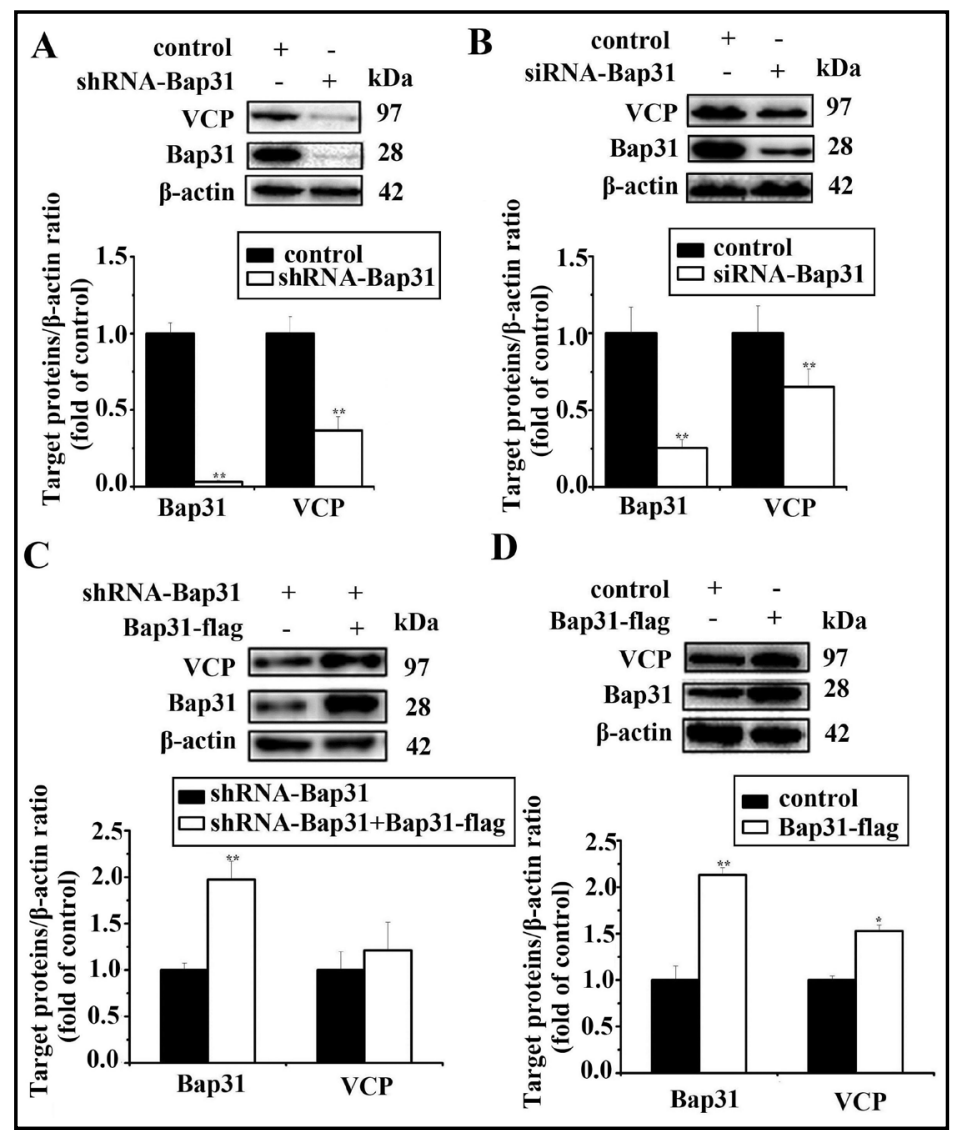

Fig. 3. Bap31 regulates the protein levels of VCP in vitro. Western blot analysis of VCP in shRNA-Bap31-transfected cells (A) and cells transfected with siRNA-Bap31 (50 nM) for $72 \mathrm{hrs}$ (B). Western blot analysis of VCP in shRNA-Bap31-transfected $\mathrm{N}_{2}$ a cells (C) and $\mathrm{N}_{2}$ a cells (D) overexpressing Bap31 (Bap31-flag) for 72 hrs. Histograms showing the relative changes in the target protein levels in Bap31 disturbed cells and control cells. $p^{*}$ vs control $<0.05, p^{* *}$ vs control $<0.01$. frontotemporal dementia, and amyotrophic lateral sclerosis [14]. Thus, Western blot analyses were used to detect the protein levels of VCP in the shRNA-Bap31- and siRNA-Bap31-transfected cells, and the VCP protein levels were reduced to $36 \%$ and $65 \%$ of those observed in the control cells, respectively (Fig. 3A and 3B). Furthermore, the protein level of VCP was downregulated by $57 \%$ (Fig. S1A) in the $\mathrm{N}_{2}$ a cells transfected with CRISPR-cas9-sgBap31 for 72 hrs (cas9Bap31).

Since the knockdown of Bap31 downregulated the expression levels of VCP, we subsequently examined whether the overexpression of Bap31 affects VCP expression. Hence, the Bap31-flag plasmid was used to overexpress Bap31 in shRNA-Bap31-transfected and nontransfected $\mathrm{N}_{2}$ a cells, and the protein levels of VCP were $120 \%$ and $160 \%$ of those in the control cells, respectively (Fig. 3C and 3D). The same results of the regulation of VCP expression by Bap31 were obtained in BHK cells (Fig. S1B and S1C). Thus, these results further confirm that Bap31 regulates VCP expression in vitro. 
Bap31 regulates the expression of VCP in Camk2a-cre Bap31/f/f mice

The hippocampus is widely used to study CNS diseases in animal models [21]. In our laboratory, Camk2a-cre Bap31 $1^{\mathrm{f} / \mathrm{f}}\left(\mathrm{Bap} 1^{-/-}\right)$and Bap31 $1^{\mathrm{f} / \mathrm{f}}\left(\mathrm{Bap} 1^{+/+}\right)$mice were bred and used to investigate whether Bap31 regulates VCP in vivo. The analysis of the results revealed that the mRNA and protein levels of Bap31 in the hippocampus of Bap31 $1^{-/-}$mice were reduced by $60 \%$ and $80 \%$ of those observed in the control mice, respectively, whereas almost no change

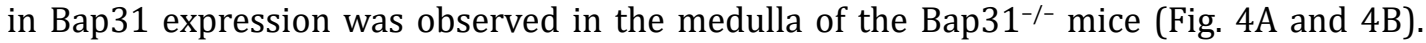
These results indicate that Camk2a-cre expression induces an efficient deletion of Bap31 in the hippocampus. Therefore, we used the hippocampus tissues of mice to investigate whether Bap31 regulates VCP expression in vivo.

The real-time PCR analysis revealed that the mRNA expression levels of VCP in the Bap31 $1^{-/-}$mice were $65 \%$ and $69 \%$ of those in the Bap31 $1^{+/+}$mice (Fig. $4 \mathrm{C}$ ) and the protein expression levels of VCP in the Bap31//- mice were $37 \%$ and $24 \%$ of those in the Bap31//+ mice (Fig. 4D). Our results further suggest that Bap31 regulates the expression of VCP at both the mRNA and protein levels in vivo.

\section{Bap31 participates in the VCP-mediated degradation of CFTR $\triangle F 508$}

Notably, both Bap31 and VCP are ERAD components [22] involved in the degradation of CFTR $\triangle F 508$. Moreover, Bap31 regulated the expression of VCP in the process of the degradation of CFTR $\Delta$ F508 (Fig. S2A and S2B). After treating the CFTR $\Delta$ F508-HA cells with cycloheximide (CHX), compared with the control cells, the protein levels of VCP were regulated by Bap31 at the zero time point (Fig. S3A and S3B). The protein stability of VCP displayed a few differences among the different groups. These differences may be due to the combination of Bap31 perturbation and CHX treatment, which may have induced the abnormal conditions in the cells or ER stress that could have affected the function of the ER.

Fig. 4. Bap31 regulates the mRNA and protein levels of VCP in vivo. Real-time PCR (A) and Western blot (B) analyses of Bap31 in the hippocampus and medulla of Bap31\% and Bap31 $1^{+/+}$ mice (16-week-old male mice). Real-time PCR (C) and Western blot (D) analyses of VCP in the hippocampus of Bap31\% and Bap31+/+ mice (16-week-old male mice). Histograms showing the relative changes in the target protein levels in Bap31\% and Bap31 $1^{+/+}$ mice. $p^{* *}$ vs control < 0.01 .

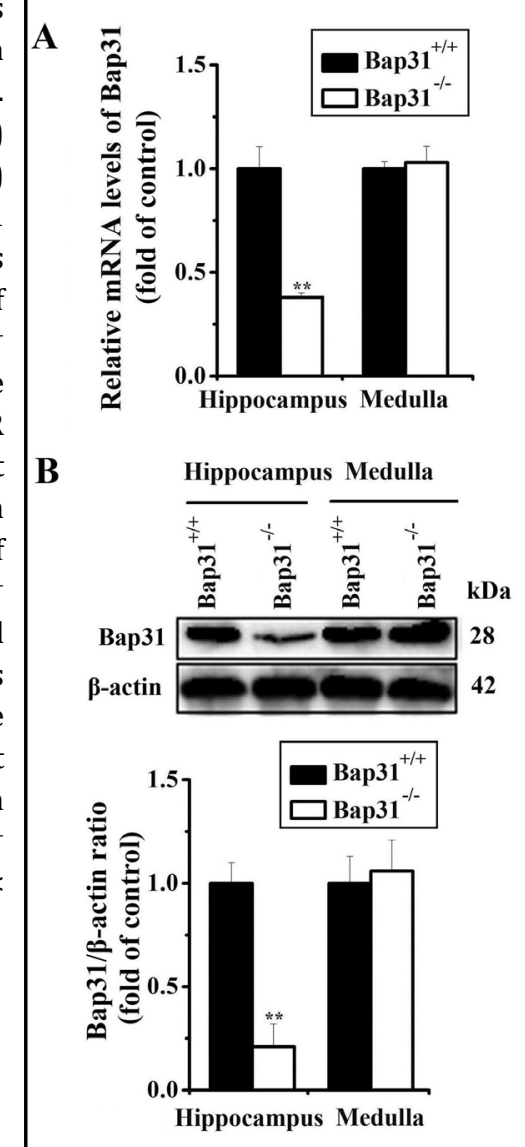

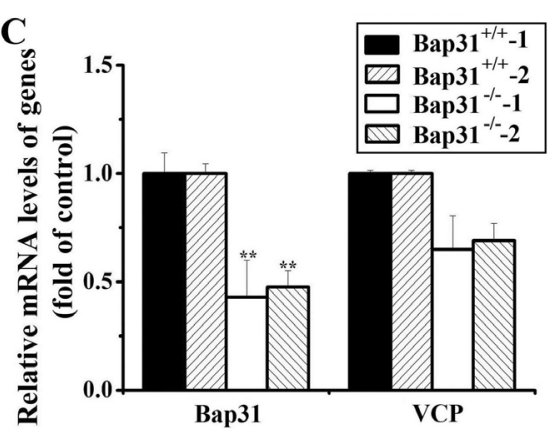

D
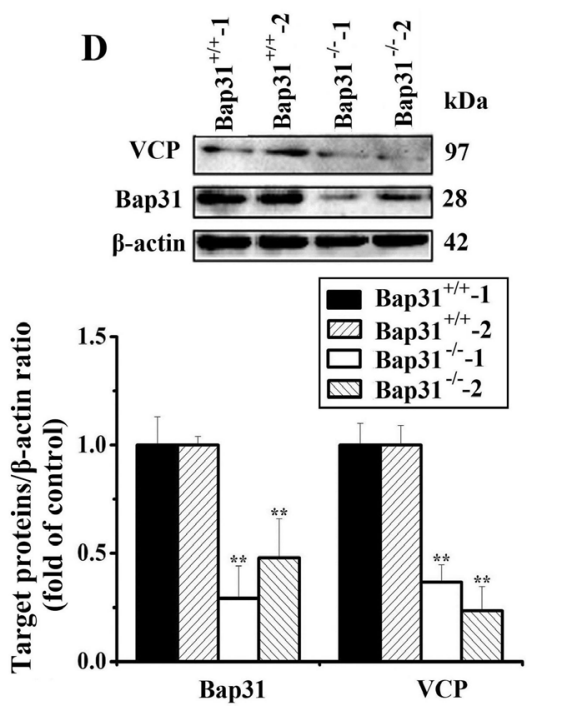


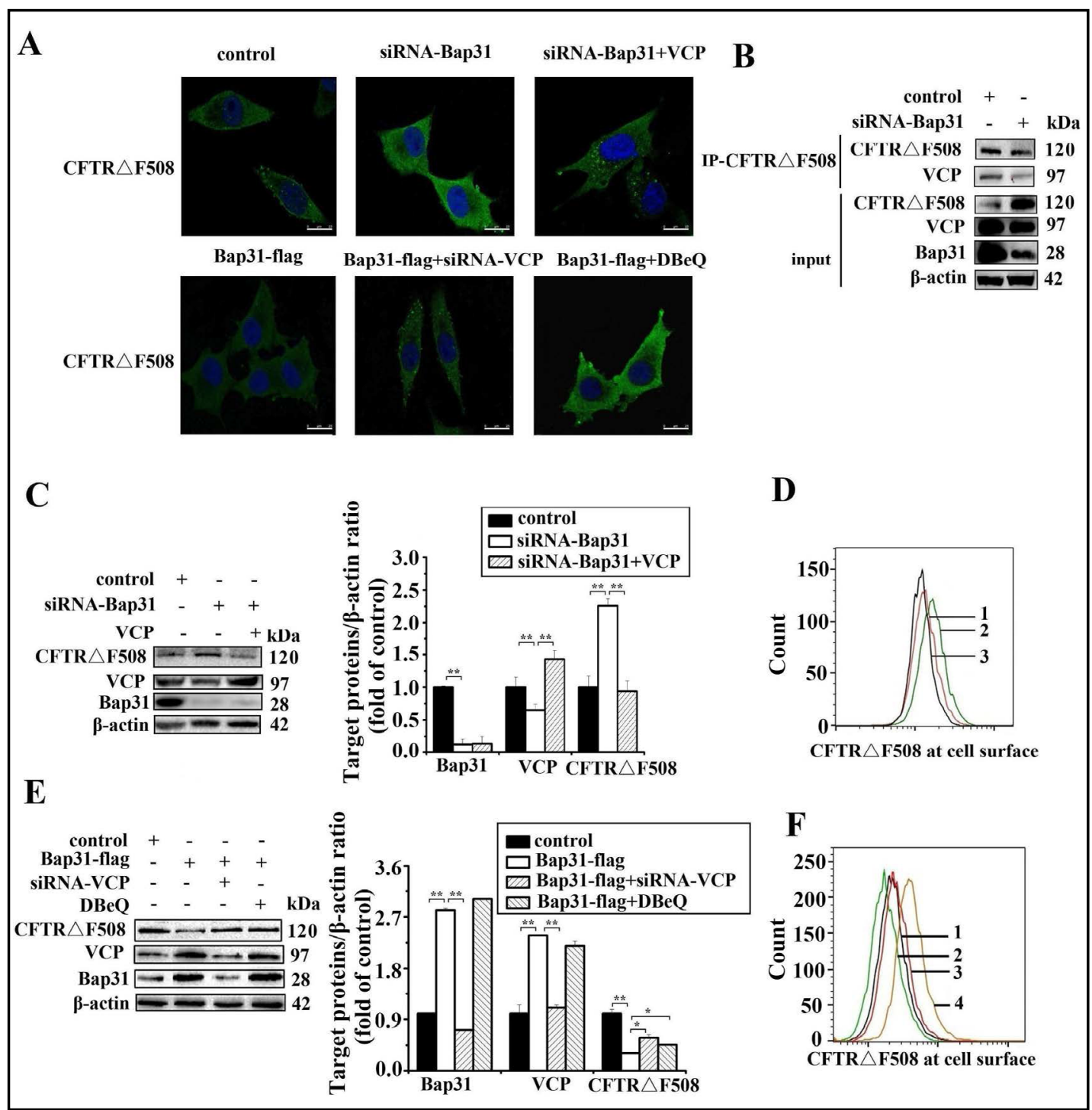

Fig. 5. Bap31 promotes CFTR $\triangle F 508$ degradation via the VCP associated pathway. In BHK cells stably expressing CFTRAF508-HA, an anti-HA antibody was used to detect the levels of CFTR $\Delta$ F508. (A) Immunofluorescence was used to detect the CFTR $\Delta$ F508 in the same groups described in B and C. The slides were stained with an anti-HA and FITC conjugated secondary antibodies to detect CFTR $\Delta$ F508 (green). The slides were stained with Hoechst 33342 to stain the nucleus (blue). Merged images of CFTR $\Delta$ F508 (green) and nucleus (blue) staining are shown. (B) Immunoprecipitation analysis of the direct interaction between CFTR F508 and VCP in Bap31 knockdown cells. (C) Western blot analysis showing the protein levels of CFTR $\Delta$ F508 in the same groups shown in D. (D) Flow cytometry analysis of cell surface CFTR $\Delta$ F508 in control cells (1), siRNA-Bap31-transfected cells (2) and siRNA-Bap31-transfected cells overexpressing VCP for $72 \mathrm{hrs} \mathrm{(3).} \mathrm{(E)} \mathrm{Western} \mathrm{blot} \mathrm{analysis} \mathrm{showing} \mathrm{the} \mathrm{protein} \mathrm{levels} \mathrm{of} \mathrm{CFTR} \Delta$ F508 in the same groups shown in F. (F) Flow cytometry analysis of cell surface CFTR $\Delta$ F508 in control cells (1), Bap31-flag overexpressing cells (2), Bap31-flag overexpressing cells transfected with siRNA-VCP for 72 hrs (3) and Bap31-flag overexpressing cells cultured with DBeQ $(2.5 \mu \mathrm{M})$ for $2 \mathrm{hrs}(4)$. Histograms showing the relative changes in the target protein levels in the samples. $p^{*}$ vs control $<0.05, p^{* *}$ vs control $<0.01$. 
The cell viabilities were reduced following treatment with $\mathrm{DBeQ}$, which is an inhibitor of VCP, at a higher concentration $(>2.5 \mu \mathrm{M})$ for 2 hrs (Fig. S4A). It has been shown that CFTR $\triangle$ F508 that escapes from degradation by the ERAD pathway can reach the cell membrane [4], therefore, we assessed CFTR $\triangle$ F508 expression on the cell surface. The results of the flow cytometry analysis showed that more CFTR $\Delta$ F508 reached the cell surface after 2 hrs of treatment with DBeQ $(2.5 \mu \mathrm{M})$ (Fig. S4B).

Immunofluorescence staining was used to detect the total levels of CFTR $\Delta$ F508. An HA antibody was used to stain the cells with altered Bap31 expression that were further treated with siRNA-VCP, VCP plasmid or DBeQ (Fig. 5A). The results indicated that the changes in VCP expression alter the degradation of CFTR $\triangle F 508$, which is targeted by Bap31. Therefore, we speculate that Bap31 regulates the degradation of CFTR $\triangle F 508$ via the ERAD process, a pathway in which VCP was involved.

To further explore this speculation, coimmunoprecipitation was used to detect the interaction between CFTR $\triangle F 508$ and VCP in Bap31 knockdown cells. Following the inhibition of Bap31 expression by siRNA treatment, the binding quantities of CFTR $\triangle F 508$ to VCP were decreased (Fig. 5B). Moreover, the overexpression of VCP in the siRNA-Bap31-transfected cells promoted the degradation of CFTR $\triangle$ F508 based on our finding that the CFTR $\Delta$ F508 protein level following VCP overexpression was $42 \%$ of that in the siRNA-Bap31-transfected cells without VCP overexpression (Fig. 5C). The knockdown of VCP by siRNA-VCP in Bap31 overexpressing cells resulted in an increase in CFTR $\Delta$ F508 protein expression to $190 \%$ of that in the Bap31 overexpressing cells (Fig. 5E). We also examined the degradation of CFTR $\Delta$ F508 in Bap31 overexpressing cells cultured with $2.5 \mu \mathrm{M}$ DBeQ. Following treatment with the VCP inhibitor, the total protein level of CFTR $\triangle$ F508 was increased to $150 \%$ of that in the control cells (Fig. 5E). Based on the determination of the cell surface expression of CFTR $\Delta$ F508 by flow cytometry (Fig. 5D and 5F), VCP affected the degradation of CFTR $\Delta$ F508 in cells with altered Bap31 expression. However, Bap31 expression was decreased for an unknown reason following the suppression of VCP expression by siRNA (Fig. 5E). Bap31 and VCP perform similar functions in cellular processes and do not directly interact with each other [22]. The regulation of Bap31 by VCP may occur via other proteins. The predicted transcription factors of Bap31 were identified based on the PROMO database (the sequences range from the upstream $2000 \mathrm{bp}$ to the downstream $100 \mathrm{bp}$ of transcription initiation site in humans). Nuclear factor kappa B subunit $1(\mathrm{NF}-\mathrm{\kappa B})$ was one of the predicted transcription factors. $\mathrm{NF}-\kappa \mathrm{B}$ can be activated by stimulation, such as by cytokines, oxidant-free radicals, etc. The activated NF- $\kappa \mathrm{B}$ translocates into the nucleus to regulate gene expression which participates in a wide variety of biological processes $[23,24]$. Vandermoere et al. reported that RNAi targeting VCP inhibits the activation of NF- $\mathrm{\kappa B}$ induced by growth factors [25]. Therefore, the regulation of VCP on Bap31 maybe occur via NF- $\kappa B$ or other Bap31 transcription factors. In addition, $\mathrm{Bcl} 2$, which is an important anti-apoptosis molecule, regulates the cell cycle by enhancing G0 and delaying the G0 to S transition. Its noted that the knockdown of VCP downregulates the protein levels of Bcl2 [26]. VCP regulates the expression levels of Bap31 maybe via the Bcl2 mediated cell cycle. Moreover, VCP and ATL1 regulate the ER and protein synthesis during the formation of dendritic spines [27]. RNAi targeting VCP may affect the function of the ER and cause a change in the expression level of Bap31. All these possibilities require further investigating. Taken together, Bap31 regulates the VCP-mediated degradation of CFTR $\Delta$ F508.

Bap31 regulates the transcription and expression of VCP through Elf2

Our results demonstrated that the VCP expression levels were regulated by Bap31 both in vivo and in vitro. We speculate that Bap31 interacts with VCP. However, a previous study indicated that there is no direct binding between Bap31 and VCP under normal conditions in NP40 buffer [22], and the same results were obtained in Chaps buffer (Fig. 6A) and under ER stress caused by serum starvation for 48 hrs (Fig. 6B) in the present study. Therefore, the regulation of Bap31 on VCP expression is not mediated through a direct interaction. 


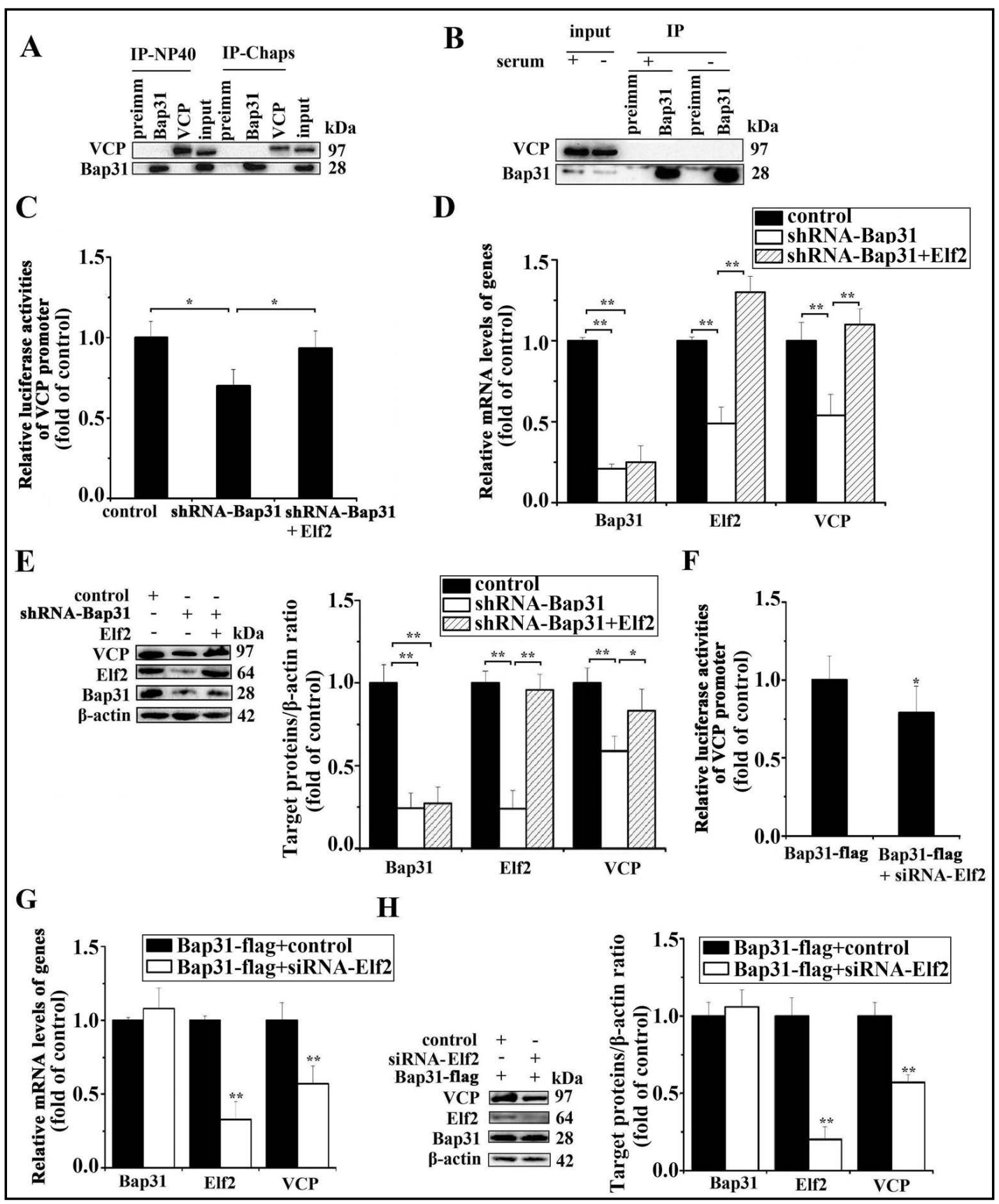

Fig. 6. Bap31 regulates the expression of VCP via Elf2. (A) Immunoprecipitation analysis of the binding between Bap31 and VCP in Chaps or NP40 buffer in $\mathrm{N}_{2}$ a cells. (B) Immunoprecipitation analysis of the interaction between Bap31 and VCP in control cells and cells that were serum starved for 48 hrs. The transcription activities of the VCP promoter (C) and the mRNA (D) and protein (E) levels of VCP in shRNABap31-transfected cells overexpressing Elf2. The transcription activities of the VCP promoter (F) and the mRNA (G) and protein (H) levels of VCP in Bap31-flag overexpressing cells transfected with siRNA-Elf2. Histograms showing the relative changes in the target protein levels in the samples. $p^{*}$ vs control $<0.05, p^{* *}$ vs control $<0.01$. 
Qiu et al. demonstrated that the transcription factor E74-like factor 2 (Elf2) regulates the expression of VCP by binding its promoter [17]. Thus, Bap31 could possibly affect the expression levels of Elf2. To explore this possibility, we detected the mRNA and protein levels of Elf2 in cells with altered Bap31 expression. The Elf2 mRNA levels in the shRNA-Bap31transfected cells and siRNA-Bap31-transfected cells were $46 \%$ and $71 \%$ of those in the control cells, respectively (Fig. S5A and S5B), and the protein levels of Elf2 in these groups were $43 \%$ and $67 \%$ of those in the control groups, respectively (Fig. S5C and S5D). These results demonstrate that Bap31 regulates Elf2 expression.

To confirm the regulation of the VCP transcription levels by Bap31, a dual luciferase assay was used to detect the transcription activities of VCP in cells with altered Bap31 expression that were further treated with siRNA-Elf2 or an Elf2 overexpression plasmid. The knockdown of Bap31 decreased the VCP promoter-based luciferase reporter activity, while the overexpression of Elf2 significantly rescued this activity (Fig. 6C), and consistent results of the effects on VCP expression were obtained with real-time PCR analyses (Fig. 6D). Following the inhibition of Elf2 by siRNA in Bap31 overexpressing cells, the VCP promoterbased luciferase reporter activity was decreased (Fig. 6F). These results support the notion that Bap31 regulates the Elf2-induced transcription activities of VCP.

Subsequently, we detected the mRNA and protein levels of VCP following the overexpression of Elf2 in shRNA-Bap31-transfected cells and found that the VCP expression levels were increased by $203 \%$ and $141 \%$ compared to those in the shRNA-Bap31-transfected cells (Fig. 6D and 6E). These results indicate that the overexpression of Elf2 restores the expression of VCP caused by Bap31 knockdown. Moreover, following the knockdown of Elf2 by siRNA-Elf2 in Bap31 overexpressing cells, the VCP mRNA and protein expression levels were $56.8 \%$ and $57 \%$ of those in the Bap31 overexpressing cells, respectively (Fig. $6 \mathrm{G}$ and $6 \mathrm{H})$. However, the mRNA levels of other genes downregulated by Bap31 were unaltered by the overexpression or knockdown of Elf2 (Fig. S6A and S6B). These results imply that Bap31 regulates VCP possibly by regulating Elf2. To clarify the mechanism through which Bap31 regulates Elf2, coimmunoprecipitation was used to detect the binding between Bap31 and Elf2, but the proteins did not form a detectable complex (Fig. S7A). This finding indicates that the regulation of Elf2 by Bap31 does not occur via direct binding. Thus, the responsible mechanisms require further investigation.

\section{Discussion}

Bap31 is mainly expressed in the ER, which is an important protein folding organelle. Bap31 is highly expressed in neurons [6] and is known to promote the transportation of multiple proteins, including newly synthesized IgD and class I major histocompatibility (MHC) molecules, to other organelles $[1,28]$. It is a fact that Bap31 mutation leads to a severe X-linked phenotype with clinical signs shared by some CNS diseases, such as congenital microcephaly and sensory neural deafness [7]. Moreover, we discovered that Bap31 is involved in the ERAD pathway [5]. Many proteins involved in CNS diseases can be degraded by the ubiquitin-proteasome pathway, which is coupled with the ERAD pathway [29]. All of these findings led us to speculate that Bap31 may be an important factor in CNS diseases.

To test this hypothesis, we used cells with stable Bap31 knockdown and cells with instantaneous Bap31 knockdown achieved via shRNA and siRNA transfection, respectively, to identify genes involved in CNS diseases that are regulated by Bap31. Thirteen genes showed consistent trends of regulation by Bap31, whereas other genes showed different trends following the altered Bap31 expression between the two groups (Fig. 1 and 2). The differences between the stable cell line and the transiently transfected cells may be explained as follows: the stable shRNA-Bap31-transfected cells existed in an abnormal state for a relatively long time, whereas the siRNA-Bap31-transfected cells did not. One of the possible explanations for how Bap31 regulates these genes is that Bap31 is an important factor in the transcription process that affects the activities of transcription factors. The detailed 
molecular mechanisms by which Bap31 affects the mRNA levels of genes involved in CNS diseases need further investigation.

We further investigated VCP, which is one of the 13 candidate genes, as a target of Bap31 in the current study. Bap31 and a VCP mutant (K524M) have been shown to interact to exert a dominant negative effect on retrotranslocation and cause proteins to accumulate at the juxtanuclear region [22]. VAMP-associated proteins (VAPs) inhibit the degradation of CFTR $\Delta$ F508 through interactions with the RMA1-Derlin-Bap31-VCP pathway [30]. In our investigation of the mechanism by which Bap31 regulates VCP expression, we first observed that Bap31 regulates the mRNA and protein expression levels of VCP both in vitro and in vitro (Fig. 3 and 4).

VCP is a component of the retrotranslocation machinery between the ER and the proteasome. Unfolded proteins are transported from the ER to the cytosol by VCP. Thereafter, these proteins are ubiquitinated by ligases. Finally, polyubiquitinated unfolded proteins are degraded by the proteasome via the interaction between VCP and Fas-associated factor 1 (FAF1) [31]. Based on this information, we speculated that Bap31 regulates the degradation of unfolded proteins partially via the VCP-associated pathway. Some newly synthesized molecules of CFTR $\triangle F 508$ become mature and reach the cell surface if they are not degraded in the ER [32]. However, more than $99 \%$ of newly synthesized CFTR $\Delta$ F508 polypeptides are degraded via the ERAD pathway; thus, few CFTR $\triangle$ F508 molecules reach the cell surface. The Western blot, immunofluorescence and flow cytometry analyses showed that Bap31 regulates the expression of VCP during the degradation of CFTR $\triangle$ F508, which is consistent with a previous study showing that Bap31 and VCP are both involved in the ERAD pathway. Moreover, a previous study demonstrated that Bap31 physically interacts with CFTR $\Delta$ F508 to promote CFTR $\Delta$ F508 degradation via the Derlin1 complex [5]. The results of the present study indicate that Bap31 regulates the expression levels of VCP, which then causes differences in the binding quantities of CFTR $\triangle F 508$ and VCP, and that VCP acts after Bap31 in the degradation of CFTR $\triangle \mathrm{F} 508$ (Fig. 5). This result suggests that Bap31 partially regulates the degradation of CFTR $\triangle F 508$ via the VCP complex.

To clarify the mechanism underlying Bap31 regulation of VCP, the direct binding between Bap31 and VCP was detected in our study. The results are consistent with a former study showing that Bap31 and VCP do not directly bind [22]. Moreover, we detected the effect of altered Bap31 expression on Elf2 expression (a transcription factor of VCP) [33] and the effect of altered Elf2 expression on VCP expression in Bap31 perturbed cells. Our results indicate that Bap31 regulates the level of Elf2 and that Elf2 modulates the transcription and expression levels of VCP (Fig. 6). Therefore, these results suggest a possible molecular mechanism through which Bap31 regulates VCP, and further research is required to confirm and further elucidate this mechanism.

Finally, we explored the mechanism of the effect of Bap31 on Elf2, and the coimmunoprecipitation results showed that Bap31 and Elf2 did not form a detectable complex. Thus, the mechanism linking Bap31 and Elf2 may involve intermediate molecules. Based on a former study, Bap31 is upstream of Derlin1 in the ERAD pathway. Therefore, Derlin1 was used to further detect the regulation of Elf2 by Bap31. The same patterns of Elf2 and VCP regulation by Bap31 and Derlin1 were observed in this study (Fig. 6 and S7). These results suggest that based on the perturbations in the same ERAD translocation pathway, this phenomenon is likely a more general response that couples retrograde components at the transcription level. Many ERAD components are involved in the degradation of CFTR $\triangle$ F508. The members of the ERAD pathway are important for promoting the degradation of abnormal proteins. If any of the members loses function in ER quality control, the excessive accumulation of misfolded or unfolded proteins occurs in the ER and causes ER stress. Thus, regulation among these pathway members may exist. Both Bap31 and VCP are ERAD components that function in the same biological processes, such as the cell cycle, ER stress, and apoptosis. For instance, cycle-related proteins (cyclin D, cyclin E1, and cyclin E2) were significantly decreased in HeLa and SiHa cells transfected with siRNA-Bap31 compared with those in the control cells. The knockdown of Bap31 arrests the cell cycle at the G0/G1 phase 


\section{Cellular Physiology Cell Physiol Biochem 2018;51:1799-1814

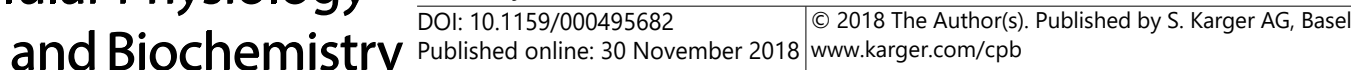 \\ Jia et al.: Bap31 Regulates VCP via Elf2}

[34]. The protein levels of cyclin D1, cyclin E, CDK2, CDK4, Ki-67 and PCNA were decreased in shRNA-VCP-transfected cells [26]. Moreover, ER stress marker levels were increased in Bap31 knockout mice after tunicamycin injection compared with those in control mice [35]. The depletion of VCP by siRNA induces ER stress and the unfolded protein response (UPR) [36]. In addition, the C-terminus of Bap31 can be cleaved by caspase 8 to generate p20, which participates in apoptosis. VCP is involved in gp130-mediated cell proliferation and anti-apoptosis [37]. The same functions are shared by Bap31 and VCP in the cell cycle, ER stress and apoptosis. The regulation of transcription levels by Bap31 may be due to this coregulation between Bap31 and VCP. Therefore, this transcriptional regulation may be very important as the role of ERAD components in the regulation of transcription has never been studied. Bap31 regulated the degradation of CFTR $\triangle$ F508 possibly through both the transcription of VCP and the retro-translocation of CFTR $\Delta$ F508. However, the detailed mechanisms and contributions remain to be clarified.

Notably, a VCP reduction can both increase the levels of tau phosphorylation at $\operatorname{Ser}^{262 / 356}[29]$ and induce mitophagy impairment and neurodegeneration in a Huntington's disease model [38]. Therefore we can study the function of Bap31 in Alzheimer's disease and Huntington's disease via its regulation of VCP. Given the diverse functions of VCP, our findings lay a foundation for future research investigating the unknown function of Bap31. In conclusion, our study finally demonstrated that Bap31 regulates VCP through its transcription factor Elf2 and partially participates in the degradation of CFTR $\Delta$ F508 through the VCP-associated pathway. The data presented here illustrate a new potential function of Bap31 in CNS diseases.

\section{Acknowledgements}

This research was supported by the National Natural Science Foundation of China (31370784, 2016YFC1302402, 31670770, U1603125 and 81473330) and the Fundamental Research Funds for the Central Universities of China (N130420004, N130120001, N141008001 and N162004003).

\section{Disclosure Statement}

The authors declare that they have no conflicts of interest.

\section{References}

$>1$ Adachi T, Schamel WW, Kim KM, Watanabe T, Becker B, Nielsen PJ, Reth M: The specificity of association of the IgD molecule with the accessory proteins BAP31/BAP29 lies in the IgD transmembrane sequence. EMBO J 1996;15:1534-1541.

-2 Ng FW, Nguyen M, Kwan T, Branton PE, Nicholson DW, Cromlish JA, Shore GC: p28 Bap31, a Bcl-2/Bcl-X and procaspase-8-associated protein in the endoplasmic reticulum. J Cell Biol 1997;139:327-338.

-3 Annaert WG, Becker B, Kistner U, Reth M, Jahn R: Export of cellubrevin from the endoplasmic reticulum is controlled by BAP31. J Cell Biol 1997;139:1397-1410.

-4 Niu KW, Xu JL, Cao YH, Hou Y, Shan M, Wang YQ Xu Y, Sun MY, Wang B: Bap31 is involved in T cell activation through TCR signal pathways. Sci Rep 2017;7:44809.

5 Wang B, Heath-Engel H, Zhang D, Nguyen N, Thomas DY, Hanrahan JW, Shore GC: BAP31 interacts with Sec61 translocons and promotes retrotranslocation of CFTR $\Delta$ F508 via the derlin-1 complex. Cell 2008;133:1080-1092.

6 Manley HA, Lennon VA: Endoplasmic reticulum membrane-sorting protein of lymphocytes (BAP31) is highly expressed in neurons and discrete endocrine cells. J Histochem Cytochem 2001;49:1235-1243. 


\section{Cellular Physiology Cell Physiol Biochem 2018;51:1799-1814 \begin{tabular}{l|l|l|l}
\hline a & DOI: 10.1159/000495682 & 2018 The Author(s). Published by S. Karger AG, Basel
\end{tabular}

7 Cacciagli P, Sutera-Sardo J, Borges-Correia A, Roux JC, Dorboz I, Desvignes JP, Badens C, Delepine M, Lathrop M, Cau P, Levy N, Girard N, Sarda P, Boespflug-Tanguy O, Villard L: Mutations in BCAP31 cause a severe X-linked phenotype with deafness, dystonia, and central hypomyelination and disorganize the Golgi apparatus. Am J Hum Genet 2013;93:579-586.

-8 Pleasure IT, Black MM, Keen JH: Valosin-containing protein, VCP, is a ubiquitous clathrin-binding protein. Nature 1993;365:459-462.

-9 Song C, Wang Q, Li CC: ATPase activity of p97-valosin-containing protein (VCP). D2 mediates the major enzyme activity, and D1 contributes to the heat-induced activity. J Biol Chem 2003;278:3648-3655.

$>10$ Dai RM, Chen E, Longo DL, Gorbea CM, Li CC: Involvement of valosin-containing protein, an atpase copurified with IkappaBalpha and $26 \mathrm{~S}$ proteasome, in ubiquitin-proteasome-mediated degradation of IkappaBalpha. J Biol Chem 1998;273:3562-3573.

11 Dai RM, Li CC: Valosin-containing protein is a multi-ubiquitin chain-targeting factor required in ubiquitinproteasome degradation. Nat Cell Biol 2001;3:740-744.

12 Ballar P, Shen Y, Yang H, Fang S: The role of a novel p97/valosin-containing protein-interacting motif of gp78 in endoplasmic reticulum-associated degradation. J Biol Chem 2006;281:35359-35368.

13 Poksay KS, Madden DT, Peter AK, Niazi K, Banwait S, Crippen D, Bredesen DE, Rao RV: Valosin-containing protein gene mutations: Cellular phenotypes relevant to neurodegeneration. J Mol Neurosci 2011;44:91102.

14 Nalbandian A, Donkervoort S, Dec E, Badadani M, Katheria V, Rana P, Nguyen C, Mukherjee J, Caiozzo V, Martin B, Watts GD, Vesa J, Smith C, Kimonis VE: The multiple faces of valosin-containing proteinassociated diseases: inclusion body myopathy with Paget's disease of bone, frontotemporal dementia, and amyotrophic lateral sclerosis. J Mol Neurosci 2011;45:522-531.

15 Yamamoto S, Tomita Y, Nakamori S, Hoshida Y, Nagano H, Dono K, Umeshita K, Sakon M, Monden M, Aozasa $\mathrm{K}$ : Elevated expression of valosin-containing protein (p97) in hepatocellular carcinoma is correlated with increased incidence of tumor recurrence. J Clin Oncol 2003;21:447-452.

-16 Tsujimoto Y, Tomita Y, Hoshida Y, Kono T, Oka T, Yamamoto S, Nonomura N, Okuyama A, Aozasa K: Elevated expression of valosin-containing protein (p97) is associated with poor prognosis of prostate cancer. Clin Cancer Res 2004;10:3007-3012.

17 Qiu Y, Morii E, Zhang B, Tomita Y, Aozasa K: E74-like factor 2 transactivates valosin-containing protein gene, a gene involved in cancer growth. Exp Mol Pathol 2008;84:226-229.

18 Morris LL, Hartman IZ, Jun DJ, Seemann J, DeBose-Boyd RA: Sequential actions of the aaa-atpase valosin-containing protein (vcp)/p97 and the proteasome $19 \mathrm{~s}$ regulatory particle in sterol-accelerated, endoplasmic reticulum (er)-associated degradation of 3-hydroxy-3-methylglutaryl-coenzyme a reductase. J Biol Chem 2014;289:19053-19066.

19 Livak KJ, Schmittgen TD: Analysis of relative gene expression data using real-time quantitative pcr and the 2(-delta delta c(t)) method. Methods 2001;25:402-408.

20 Bayes A, van de Lagemaat LN, Collins MO, Croning MD, Whittle IR, Choudhary JS, Grant SG: Characterization of the proteome, diseases and evolution of the human postsynaptic density. Nat Neurosci 2011;14:19-21.

$\checkmark 21$ Moodley KK, Chan D: The hippocampus in neurodegenerative disease. Front Neurol Neurosci 2014;34:95108.

-22 Wakana Y, Takai S, Nakajima K, Tani K, Yamamoto A, Watson P, Stephens DJ, Hauri HP, Tagaya M: Bap31 is an itinerant protein that moves between the peripheral endoplasmic reticulum (ER) and a juxtanuclear compartment related to ER-associated Degradation. Mol Biol Cell 2008;19:1825-1836.

-23 Ma J, Liu C, Yang Y, Yu J, Yang J, Yu S, Zhang J, Huang L, C/EBP $\beta$ acts ppstream of NF- $\kappa B$ p65 subunit in OxLDL-induced IL-1 $\beta$ production by macrophages. Cell Physiol Biochem. 2018;48:1605-1615.

-24 Mishra V, Banga J, Silveyra P: Oxidative stress and cellular pathways of asthma and inflammation: Therapeutic strategies and pharmacological targets. Pharmacol Ther 2018;181:169-182.

-25 Vandermoere F, El Yazidi-Belkoura I, Slomianny C, Demont Y, Bidaux G, Adriaenssens E, Lemoine J, Hondermarck $\mathrm{H}$ : The valosin-containing protein ( $\mathrm{vcp}$ ) is a target of akt signaling required for cell survival. J Biol Chem 2006;281:14307-14313.

-26 Fu Q, Jiang Y, Zhang D, Liu X, Guo J, Zhao J: Valosin-containing protein (vcp) promotes the growth, invasion, and metastasis of colorectal cancer through activation of stat3 signaling. Mol Cell Biochem 2016;418:189198. 


\section{Cellular Physiology Cell Physiol Biochem 2018;51:1799-1814

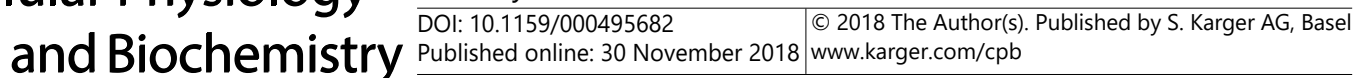 \\ Jia et al.: Bap31 Regulates VCP via Elf2}

27 Shih YT, Hsueh YP: Vcp and atl1 regulate endoplasmic reticulum and protein synthesis for dendritic spine formation. Nat Commun 2016;7:11020.

28 Ladasky JJ, Boyle S, Seth M, Li H, Pentcheva T, Abe F, Steinberg SJ, Edidin M: Bap31 enhances the endoplasmic reticulum export and quality control of human class I MHC molecules. J Immimol 2006;177:6172-6181.

29 Abisambra JF, Jinwal UK, Blair LJ, OLeary JC 3rd, Li Q, Brady S, Wang L, Guidi CE, Zhang B, Nordhues BA, Cockman M, Suntharalingham A, Li P, Jin Y, Atkins CA, Dickey CA: Tau accumulation activates the unfolded protein response by impairing endoplasmic reticulum-associated degradation. J Neurosci 2013;33:94989507.

-30 Ernst WL, Shome K, Wu CC, Gong X, Frizzell RA, Aridor M: VAMP-associated proteins (VAP) as Receptors That Couple CysticFibrosis Transmembrane Conductance Regulator (CFTR) Proteostasis with Lipid Homeostasis. J Biol Chem 2016;291:5206-5220.

-31 Lee JJ, Park JK, Jeong J, Jeon H, Yoon JB, Kim EE, Lee KJ: Complex of Fas-associated factor 1 (FAF1) with valosin-containing protein (VCP)-Npl4-Ufd1 and polyubiquitinated proteins promotes endoplasmic reticulum-associated degradation (ERAD). J Biol Chem 2013;288:6998-7011.

32 Ballar P, Ors AU, Yang H, Fang S: Differential regulation of CFTRDeltaF508 degradation by ubiquitin ligases gp78 and Hrd1. Int J Biochem Cell Biol 2010;42:167-173.

33 Zhang B, Tomita Y, Qiu Y, He J, Morii E, Noguchi S, Aozasa K: E74-like factor 2 regulates valosin-containing protein expression. Biochem Bioph Res Co 2007;356:536-541.

-34 Dang E, Yang S, Song C, Jiang D, Li Z, Fan W, Sun Y, Tao L, Wang J, Liu T, Zhang C, Jin B, Wang J, Yang K: Bap31, a newly defined cancer/testis antigen, regulates proliferation, migration, and invasion to promote cervical cancer progression. Cell Death Dis 2018;9:791.

-35 Wu Z, Yang F, Jiang S, Sun X, Xu J: Induction of liver steatosis in bap31-deficient mice burdened with tunicamycin-induced endoplasmic reticulum stress. Int J Mol Sci 2018;19:2291.

-36 Bastola P, Neums L, Schoenen FJ, Chien J: Vcp inhibitors induce endoplasmic reticulum stress, cause cell cycle arrest, trigger caspase-mediated cell death and synergistically kill ovarian cancer cells in combination with salubrinal. Mol Oncol 2016;10:1559-1574.

-37 Shirogane T, Fukada T, Muller JM, Shima DT, Hibi M, Hirano T: Synergistic roles for pim-1 and c-myc in stat3-mediated cell cycle progression and antiapoptosis. Immunity 1999;11:709-719.

-38 Guo X, Sun X, Hu D, Wang YJ, Fujioka H, Vyas R, Chakrapani S, Joshi AU, Luo Y, Mochly-Rosen D, Qi X: VCP recruitment to mitochondria causes mitophagy impairment and neurodegeneration in models of Huntington's disease. Nat Commun 2016;7:12646. 